\title{
Le développement de l'agriculture biologique : effets directs et indirects dans le monde agricole et non-agricole. Une enquête en île-de-France
}

The development of organic farming: direct and indirect impacts in the agricultural world and the non-agricultural world. A survey in the Paris region.

\section{Aurélie Cardona}

\section{OpenEdition}

Journals

Édition électronique

URL : http://journals.openedition.org/economierurale/4292

DOI : 10.4000/economierurale.4292

ISSN : 2105-2581

\section{Éditeur}

Société Française d'Économie Rurale (SFER)

Édition imprimée

Date de publication : 20 janvier 2014

Pagination : 183-194

ISSN : 0013-0559

Référence électronique

Aurélie Cardona, «Le développement de l'agriculture biologique : effets directs et indirects dans le monde agricole et non-agricole. Une enquête en Île-de-France », Économie rurale [En ligne], 339-340 | janvier-mars 2014, mis en ligne le 20 janvier 2016, consulté le 01 mai 2019. URL : http:// journals.openedition.org/economierurale/4292; DOI : 10.4000/economierurale.4292 


\title{
Le développement de l'agriculture biologique : effets directs et indirects dans le monde agricole et non agricole Une enquête en Île-de-France
}

\author{
Aurélie CARDONA • INRA, UR 767 Écodéveloppement, Avignon \\ aurelie.cardona@avignon.inra.fr
}

Si les surfaces converties à l'agriculture biologique restent faibles en France, ce mode de production s'est développé au cours des dernières décennies. Les politiques de soutien à la conversion, l'organisation de la filière et la multiplication des crises sanitaires y ont largement contribué. Dans ce contexte et sur la base d'une enquête réalisée en Île-de-France, cet article se donne pour objectif d'analyser les effets du développement de ce mode de production au sein du monde agricole et du monde non agricole. Il s'agira de montrer en quoi la diffusion de l'agriculture biologique et de ses techniques constitue un aiguillon pour l'écologisation des pratiques agricoles ; mais aussi d'expliquer comment des acteurs non agricoles peuvent s'appuyer sur la défense de l'agriculture biologique, désormais bien connue du grand public, pour revendiquer l'écologisation de leurs territoires.

MOTS-CLÉS : agriculture biologique, monde agricole, acteurs non agricoles, transition

\section{The development of organic farming: direct and indirect impacts in the agricultural world and the non-agricultural world. A survey in the Paris region}

If the areas converted to organic farming remain low in France, they are increasing during the last decades. The policies supporting organic conversion, the organization of the organic sector and the food scares have contributed to the development of organic farming. In this context and on the basis of a survey conducted in the Paris region, this paper aims to analyze the effects of the development of organic farming in the agricultural and non-agricultural world. It shows how the spread of organic farming and its techniques can contribute to an ecologization of farming practices. This paper aims also to explain how non-farming people choose to support organic farming to favor the ecologization of their living environment. (JEL: Q01-Q15)

KEYWORDS: organic farming, agricultural world, non-farming people, transition

L agriculture biologique désigne un ensemble de pratiques agricoles, définies par des agriculteurs, des scientifiques, des écologistes et des consommateurs contre le mode de production dominant de l'agriculture, dit « conventionnel » basé sur l'usage d'intrants de synthèse en vue d'une productivité maximale ${ }^{1}$ (Michelsen, 2001 ;

1. Intrants de synthèse désigne ici les engrais chimiques (azote) utilisés pour fertiliser les sols et les produits phytosanitaires (ou pesticides) chimiques employés pour détruire les organismes nuisibles aux cultures, parmi lesquels on trouvera les insecticides, les fongicides, les herbicides (par exemple le Round up).
Van Dam, Nizet et al., 2009). Si elle a été reconnue officiellement en France par la Loi d'orientation agricole (LOA) de 1980 et son cahier des charges homologué par les pouvoirs publics en 1986, elle a longtemps été perçue comme marginale et mal connue dans la mesure où elle désigne des pratiques diverses. Portée par des mouvements hétérogènes (Le Pape et Rémy, 1988 ; Leroux, 2011), elle ne concerne qu'un petit nombre d'agriculteurs et ne représente qu'une part minime de la production agricole française.

Cependant, au fil des dernières décennies, avec le développement de techniques 
agricoles de plus en plus créatrices d'incertitudes (farines animales, OGM, pesticides...), les critiques envers l'agriculture conventionnelle n'ont cessé de croître. Dans ce contexte, l'agriculture biologique telle que définie par son cahier des charges a peu à peu acquis une certaine reconnaissance en tant qu'alternative à l'agriculture conventionnelle. La diffusion des produits alimentaires biologiques se développe et les surfaces agricoles certifiées en agriculture biologique augmentent régulièrement - elles atteignent aujourd'hui 3,5\% de la $\mathrm{SAU}^{2}$. La distribution de produits biologiques par les grandes et moyennes surfaces (GMS) et la multiplication des politiques publiques visant à soutenir l'agriculture biologique contribuent à cette progression et à une forme d'institutionnalisation de ce mode de production (Piriou, 2002). Certes, les surfaces certifiées en agriculture biologique sont encore faibles en France par rapport à d'autres pays européens, néanmoins on peut s'interroger sur les effets directs et indirects du développement de ce mode de production sur le monde agricole conventionnel, mais aussi dans le monde non agricole.

Notre questionnement s'appuie sur l'étude de trois terrains franciliens, analysés dans le cadre d'un travail de thèse, au cours duquel l'interrogation porte plus particulièrement sur le rôle des acteurs non agricoles $^{3}$ dans les transitions vers

2. Chiffres de l'Agence Bio - Édition 2012. Disponible sur : http://www.agencebio.org/sites/default/ files/upload/documents/4_Chiffres/BrochureCC/ CC2012_Chap4_1_Prod.pdf

3. «Non agricoles » désigne ici les acteurs extérieurs au «monde agricole », plus précisément des organismes impliqués dans la gestion de l'eau, des personnes d'associations environnementales, des habitants, des consommateurs... Néanmoins, cette catégorisation est à nuancer. D'une part parce que ces acteurs peuvent avoir des liens personnels au monde agricole. D' autre part parce que, dès qu'ils tissent des liens avec des agriculteurs et interviennent en faveur de changements de pratiques agricoles, il devient difficile de les considérer comme extérieurs au «monde agricole ». l'agriculture bas intrants et l'agriculture biologique (Cardona, 2012). Le premier terrain se situe en Seine-et-Marne. Il s'agit d'un bassin versant où à la suite de problèmes de quantité et de qualité de l'eau, un comité d'usagers de la nappe - transformé par la suite en association - a été formé afin d'assurer un suivi et une gestion de la nappe phréatique qui assure en grande partie l'approvisionnement en eau de l'agglomération parisienne. L'association se veut indépendante des pouvoirs publics, des industriels et des organismes gestionnaires de l'eau. On s'est plus particulièrement intéressé ici aux animations et actions développées par cette association en direction du monde agricole et qui ont pour objectif de réduire la teneur de l'eau en produits phytosanitaires. Des entretiens ont été réalisés auprès de céréaliers situés sur un bassin versant concerné par les animations de l'association, auprès de membres de l'association, mais également auprès du personnel de la Chambre d'agriculture de Seine-et-Marne et des conseillers techniques des organismes stockeurs (coopératives, négociants) présents sur la zone. Les interactions de ces acteurs lors de réunions ou formations aux techniques à bas niveaux d'intrants ou biologiques ont également été observées. Le deuxième terrain situé en Essonne prend forme autour d'un conflit d'aménagement. Ici, des acteurs locaux se mobilisent pour le développement d'une agriculture " propre » et de proximité afin de valoriser un espace menacé d'urbanisation par un projet d'aménagement de l'État. Ce terrain a été traité à travers la réalisation d'entretiens auprès des agriculteurs (majoritairement des céréaliers même si certains pratiquent également le maraîchage ou l'élevage), des acteurs institutionnels (issus des mairies et Chambres d'agriculture) et des acteurs issus de la société civile. Un suivi de divers moments collectifs a également été réalisé afin de mieux comprendre la nature 
des interactions entre ces acteurs. Le dernier terrain se situe dans les Yvelines et concerne un conflit d'aménagement où s'opposent des partisans de la construction d'un circuit de Formule 1 et des acteurs mobilisés en faveur de l'installation d'exploitations pratiquant l'agriculture biologique ou engagées à mettre en œuvre à l'avenir ce mode de production. Il a été abordé à travers la constitution d'un corpus de presse et avec l'aide d'un logiciel d'analyse textuelle ${ }^{4}$. Parallèlement à ces terrains, dix entretiens auprès de céréaliers biologiques franciliens ont été réalisés afin de comprendre quels ont été leurs cheminements en termes de changements de pratiques techniques, mais aussi l'évolution de leurs conceptions au fil de leur parcours professionnel.

Sur la base de ces trois terrains, on s'intéressera dans un premier temps au rôle que peut jouer l'agriculture biologique dans une forme d'écologisation de l'agriculture, c'est-à-dire l'intégration croissante des enjeux environnementaux (Mélard, 2008). Puis, on considérera les effets du développement de l'agriculture biologique dans le monde non agricole.

\section{Le développement de l'agriculture biologique, pour une écologisation des pratiques agricoles?}

Dans un premier temps, nous questionnerons les conséquences du développement de l'agriculture biologique dans le monde agricole à travers en considérant l'évolution de l'image de ce mode de production, puis les changements des pratiques agricoles qui en découlent.

4. Le logiciel d'analyse textuelle est Prospéro, élaboré au sein du Groupe de sociologie pragmatique et réflexive (GSPR), laboratoire de l'École des Hautes Études en Sciences Sociales (EHESS).

\section{Une perception plus positive de l'agriculture biologique dans le monde agricole}

Depuis l'homologation du cahier des charges de l'agriculture biologique en France, la perception de ce mode de production a évolué. Elle est mieux connue et reconnue par les agriculteurs et les institutions agricoles conventionnelles. Cette évolution est notamment le résultat de l'augmentation des surfaces certifiées en agriculture biologique qui constitue bien un changement - certes encore minoritaire - dans le paysage agricole français. Selon les chiffres de l'Agence Bio, en 1995 , on comptait 3602 exploitations engagées en agriculture biologique en France ; en 2000, 8985 exploitations ; en 2005, 11402 ; et enfin en 2010, 19594 exploitations utilisaient ce mode de production.

Cette progression du nombre d'exploitations a tout d'abord contribué à « donner à voir » au sens propre du terme ce qu'était l'agriculture biologique à des agriculteurs conventionnels - ce qui a pu en inciter certains à s'y convertir. Pour un certain nombre d'agriculteurs, « l'exemplarité » constitue l'une des meilleures façons de se renseigner sur une technique avant d'envisager de l'adopter. C'est dans cette perspective que sont organisés les «tours de plaine » consistant en des visites d'essais ou de parcelles chez des agriculteurs lorsqu'il s'agit de diffuser de nouvelles pratiques. Ainsi, l'observation de parcelles d'exploitations voisines conduites selon les principes de l'agriculture biologique, montrant que ce système peut fonctionner et être suffisamment productif, peut constituer un « dispositif de preuve » passant par l'expérience sensible (Bessy et Chateauraynaud, 1995). La diffusion de connaissances à travers la multiplication des exploitations biologiques a ainsi contribué à entretenir la dynamique de progression du nombre de conversions comme le montrent les propos de cet agriculteur aujourd'hui certifié en agriculture biologique : 
«On est pas mal à en faire dans le coin... avec un agriculteur proche, lui, ça faisait 4 ans qu'il était en bio, donc on avait un peu de recul quand même pour voir comment ça pouvait marcher. Il est en céréales et bon ça marche, donc ça m'a aidé à la conversion, c'était facile....Ben oui parce qu'on a du recul, on a les mêmes terres» (Agriculteur biologique).

Le développement de l'agriculture biologique a également contribué à une meilleure reconnaissance des pratiques de ce mode de production et de leur technicité par les agriculteurs conventionnels. Pendant longtemps, les pratiques de l'agriculture biologique ont souffert d'un discrédit important. Pour un certain nombre d'agriculteurs, l'impossibilité d'utiliser des solutions chimiques pour le désherbage signifiait un retour à des techniques archaïques telles l'usage de la «binette » fréquemment évoqué par les maraîchers, ou l'arrachage des chardons « à la main » pour les céréaliers. Or, avec la multiplication des exploitations, la connaissance des pratiques de l'agriculture biologique s'est diffusée et il est apparu que leur maîtrise demandait une certaine technicité de la part des agriculteurs. C'est notamment le cas avec l'usage de la houe rotative ou la herse étrille :

"[La herse étrille] ce n'est pas la peine de la sortir si on n'a pas les conditions [et il faut] adapter la culture à la herse étrille, c'est-à-dire un peu plus de densité, parce que sinon vous allez faire sauter des plantes [...] il faut apprendre à s'en servir et je pense que ce n'est pas en une année qu'on apprend à s'en servir » (Agriculteur conventionnel).

En outre, devant l'expansion de ce mode de production, des outils mécaniques très pointus se sont récemment développés tels que la bineuse autoguidée qui distingue d'elle-même les adventices de la culture en place, et cible ainsi le désherbage au centimètre près grâce à une caméra. Pour un certain nombre d'agriculteurs, cet outil particulièrement technique signe définitivement l'entrée de l'agriculture biologique dans la modernité :

«On est passé sur de la haute technologie, ça, ça n'est pas la culture de grandpère!»(Agriculteur biologique).

Cette technicisation et sa vulgarisation - au sens propre du terme - contribuent à faire évoluer la perception de l'agriculture biologique en mettant à mal l'a priori d'archaïsme dont elle est souvent victime.

Parallèlement à la diffusion de connaissances sur l'agriculture biologique favorisant une meilleure reconnaissance chez les agriculteurs conventionnels, on constate également une transformation au sein des institutions politiques et professionnelles agricoles traditionnelles. D'abord, on observe une évolution de la prise en charge et du soutien à l'agriculture biologique au sein de la politique agricole. Cela se traduit dans un premier temps par la mise en œuvre d'aides publiques soutenant son développement comme la Mesure agri-environnementale (MAE) «Conversion à l'agriculture biologique » (CAB) instaurée depuis 1992 au niveau européen. D'autres aides ont par la suite été créées comme l'aide « Maintien de l'agriculture biologique » (MAB).

Ensuite, au sein des structures d'encadrement professionnelles agricoles, la perception de ce mode de production a également changé au fil du temps. Ainsi, en Seine-et-Marne, on a pu noter une appropriation progressive de ce mode de production par la Chambre d'agriculture, même si le conseil agricole en agriculture biologique a longtemps été considéré comme «à part »-selon les mots d'un des employés de cet organisme. En 1983, un conseiller technique décide de dédier une partie de son temps à l'agriculture biologique. La présidence et la direction de la 
Chambre d'agriculture acceptent cette situation « tant que [son] travail n'en souffre pas ». Cependant au fil des ans, la demande de conseil de la part des agriculteurs s'accroît et suite à l'embauche d'un nouveau conseiller technique conventionnel pour le remplacer en 1995, il peut consacrer la totalité de son temps de travail à l'agriculture biologique. Il faut noter que ce conseiller est alors rattaché physiquement au siège du Groupement des agriculteurs biologiques d'Île-de-France (GAB IDF) à environ $70 \mathrm{~km}$ du siège de la Chambre d'agriculture, bien que demeurant un membre du personnel de la Chambre d'agriculture de Seine-et-Marne. Il a d'ailleurs monté à ce moment-là un programme financé par la Région Île-de-France ayant pour objectif le développement de l'agriculture biologique et dont le GAB IDF est le bénéficiaire direct et le coordonnateur. $\mathrm{Ce}$ programme assure également alors une partie de son salaire - en ce sens, le conseil en agriculture biologique se trouve toujours « à part » d'un point de vue budgétaire. Dans le cadre de ce programme soutenu par une politique régionale forte, on assiste à un développement du conseil et de l'accompagnement pour l'agriculture biologique en Seine-et-Marne, notamment avec l'embauche d'un nouveau conseiller. Dans cette perspective, le budget est réparti plus équitablement entre le GAB IDF et la Chambre d'agriculture et celle-ci en vient finalement à « rapatrier » ses conseillers à son siège, afin de bien se faire identifier comme acteur incontournable de l'agriculture biologique. Ses conseillers techniques en agriculture biologique sont en effet souvent confondus avec le personnel du GAB

5. Depuis 1988, le Groupement des agriculteurs biologiques de la Région Île-de-France (GAB IDF) rassemble les producteurs franciliens certifiés en agriculture biologique ou en conversion. Le GAB IDF est membre du réseau des Groupements régionaux d'agriculteurs biologiques (GRAB), coordonné à l'échelle nationale par la Fédération nationale des agriculteurs biologiques (FNAB).
IDF. Ainsi aujourd'hui, la Chambre d'agriculture de Seine-et-Marne compte deux conseillers techniques en agriculture biologique et la reconnaissance de ce mode de production semble acquise alors même que ce département est dominé par la grande culture céréalière conventionnelle. Ce constat peut avoir ses conséquences pour le monde agricole dans son ensemble.

\section{L'agriculture biologique, aiguillon pour une agriculture plus respectueuse de l'environnement}

Le développement de l'agriculture biologique conduit à sa connaissance et reconnaissance croissantes dans le monde agricole. Dans la lignée de travaux récents (Lamine et Bellon, 2009), on peut penser que la diffusion de l'agriculture biologique contribue également à la diffusion de modes de production basés sur un usage limité des intrants de synthèse et donc potentiellement plus respectueux de l'environnement. De fait, l'analyse des terrains franciliens sur lesquels se base cet article montre que l'augmentation du nombre d'exploitations en agriculture biologique, l'accroissement de la demande en produits biologiques, la diffusion des techniques biologiques, une meilleure prise en charge par les systèmes d'encadrement et surtout l'évolution de la perception de l'agriculture biologique engendrent le développement de pratiques agricoles moins consommatrices en intrants que les pratiques conventionnelles.

Celles-ci sont d'abord le résultat de la multiplication des échanges entre les agriculteurs pratiquant l'agriculture biologique et ceux ne la pratiquant pas : soit de manière informelle compte tenu de l'évolution des perceptions, soit de manière plus formelle à travers, par exemple, l'organisation de formations à l'agriculture biologique à destination des agriculteurs conventionnels, comme c'est le cas à la Chambre d'agriculture de Seine-et-Marne. 
Ainsi, la diffusion de la connaissance sur les outils mécaniques de l'agriculture biologique permet à certains agriculteurs d'envisager leur utilisation sur des exploitations conventionnelles. Dans ce contexte, les agriculteurs ont de plus en plus recours à des formes d' « hybridation technique » (Ansaloni et Fouilleux, 2006) combinant plusieurs référentiels tels que la modulation de dose, les techniques de cultures simplifiées, la production intégrée, mais aussi l'agriculture biologique, afin de réduire leur usage d'intrants de synthèse. Ils parviennent ainsi à bricoler des «pratiques intermédiaires » qui se situent entre l'agriculture conventionnelle et l'agriculture biologique (Cardona, Lamine et al., 2012) qui ne nécessitent pas une totale reconception du système de production (Hill et MacRae, 1996). Ainsi, dans les cas étudiés où des acteurs non agricoles se mobilisent en faveur du développement d'une agriculture plus respectueuse de l'environnement, des «pratiques intermédiaires » sont mises en place par un certain nombre d'agriculteurs sans pour autant bouleverser le système de culture, comme cela peut être le cas lors d'une conversion à l'agriculture biologique. Par exemple, un nombre croissant de céréaliers conventionnels utilisent désormais des outils de désherbage mécanique en complément ou pour remplacer le désherbage chimique. On assiste bien au développement de nouvelles pratiques agricoles moins consommatrices en intrants de synthèse et qu'on peut supposer plus respectueuses de l'environnement que les pratiques conventionnelles.

Néanmoins, il est important de noter que ces «pratiques intermédiaires » ne se construisent pas contre l'agriculture conventionnelle, mais constituent davantage des ajustements de celle-ci pour faire face à des contraintes croissantes. La mise en évidence de nombreuses pollutions causées par l'utilisation d'intrants de synthèse pour la fertilisation des cultures ou la protection contre les maladies et ravageurs, a conduit de nombreux organismes et notamment les gestionnaires de l'eau à préconiser la réduction, voire l'interdiction de certains intrants de synthèse. Ce fut par exemple le cas en 2003 en France, avec l'interdiction des triazines, une famille de produits phytosanitaires utilisés comme désherbants. Ayant vécu ce précédent, un certain nombre d'agriculteurs modifient leurs pratiques pour anticiper d'autres interdictions potentielles (Lamine, 2011). Les populations non agricoles sont également de mieux en mieux informées sur les dangers de l'usage des intrants de synthèse en agriculture pour la santé et l'environnement et ont de fortes attentes envers l'agriculture. Les fluctuations du cours des céréales dans le secteur conventionnel et la hausse des prix des intrants de synthèse pouvant réduire la marge brute touchée par les agriculteurs constituent également une contrainte importante. Depuis le début des années 1990, un certain nombre d'agriculteurs cherche à réduire leurs charges au maximum, notamment en réduisant leur utilisation d'intrants de synthèse.

Le développement de ces « pratiques intermédiaires » mêlant pratiques conventionnelles et pratiques issues de l'agriculture biologique, s'inscrit donc dans une perspective d'ajustement aux contraintes environnementales et économiques croissantes, et non pas dans une logique de contestation de l'agriculture conventionnelle. Pour ces agriculteurs, souvent en fin ou en début de carrière (moments où les prises de risques sont limitées), des changements sont effectués, mais ils restent mineurs. Cette dynamique fait écho et est en partie rendue possible par les processus d'affaiblissement du ressort contestataire de l'agriculture biologique (Leroux, 2011), dans un contexte de « conventionalisation » de ce mode de production de plus en plus structuré autour de contraintes économiques portées par des grands groupes agro-alimentaires à la manière de l'agriculture conventionnelle (Guthman, 2004). Si pendant longtemps 
la pratique de l'agriculture biologique fut exclusivement considérée comme « une critique en acte des systèmes de production, donc une interrogation de fond sur le sens de la pratique moderniste » (Rémy, 1989), elle constitue aujourd'hui de plus en plus un mode de production parmi d'autres, dont des agriculteurs conventionnels peuvent aisément emprunter les techniques sans pour autant nécessairement adhérer à ses valeurs fondatrices. Cela peut largement favoriser la reprise ou l'adoption de ses techniques par des agriculteurs conventionnels et ainsi contribuer à l'élaboration d'autres formes d'agricultures plus respectueuses de l'environnement que le modèle conventionnel dominant.

Au sein du monde agricole, le développement de l'agriculture biologique a un certain impact. Si certains mettent en doute la pérennité économique, agronomique et environnementale de l'utilisation de techniques de l'agriculture biologique dans une exploitation conventionnelle; d'autres mettent en place des pratiques agricoles plus respectueuses de l'environnement inspirées de l'agriculture biologique : des « pratiques intermédiaires ». Cela n'a été rendu possible que parce que l'agriculture biologique est de mieux en mieux connue et reconnue, mais aussi parce qu'elle devient un mode de production parmi d'autres dans un contexte d'affaiblissement de sa dimension contestataire. Au-delà de ces transformations, on peut se demander si la diffusion de l'agriculture biologique ne peut pas entraîner d'autres changements, en dehors du monde agricole.

\section{Le développement de l'agriculture biologique, pour une écologisation de la société ?}

Le développement de l'agriculture biologique et ses effets ne concernent pas seulement le monde agricole. Cela va de soi puisqu'il a toujours été en partie porté par des acteurs extérieurs au monde agricole qu'ils soient philosophes, consommateurs ou militants environnementalistes. Néanmoins, pendant longtemps ces acteurs non agricoles intéressés au développement de l'agriculture biologique et souvent fondateurs d'organisations ou mouvements sociaux visant à promouvoir ce mode de production ont pu sembler marginaux. Avec l'institutionnalisation de l'agriculture biologique, sa diffusion dans de nombreux circuits commerciaux et le fait qu'elle soit de mieux en mieux connue du grand public, on peut se demander si cela ne favorise pas l'émergence de nouveaux phénomènes sociaux.

\section{Argument et outil dans les mobilisations collectives}

Une des premières conséquences du succès de l'agriculture biologique et de la communication sur ce mode de production, son cahier des charges et son label, est l'identification de l'agriculture biologique par de nombreux acteurs non agricoles comme la seule alternative à l'agriculture conventionnelle. On a pu notamment le constater sur les terrains franciliens et les agriculteurs en sont bien conscients : «C'est soit on met des produits et on est tout mauvais, soit on est bio et on est tout bon » (Agriculteur conventionnel).

Dans les conceptions de la majorité des acteurs du monde non agricole, peu de place est laissée en effet pour les «pratiques intermédiaires » évoquées précédemment et observées chez les agriculteurs. Cependant, cette identification claire et précise de l'agriculture biologique comme alternative à l'agriculture conventionnelle est sans doute ce qui permet à des acteurs non agricoles de se mobiliser et d'agir en faveur de changements de pratiques agricoles, comme c'est le cas dans les terrains observés. Ils sont en mesure d'exiger des changements auprès d'agriculteurs conventionnels parce qu'ils connaissent une alternative : l'agriculture biologique. 
Par ailleurs, la diffusion de l'information sur l'agriculture biologique et son développement conduisent les acteurs à utiliser la défense de l'agriculture biologique comme un argument lors d'actions collectives. Dans les cas analysés, il apparait que pour contrer un projet d'aménagement urbain et donc une menace faite à leur cadre de vie - composé ici d'espaces agricoles - certains acteurs se sont mobilisés en faveur de la conversion à l'agriculture biologique de terres agricoles, pour légitimer leur préservation. Se mobiliser pour l'agriculture biologique peut également permettre de protéger des biens publics tels que le paysage ou l'eau. Or le recours à ces biens dans une mobilisation peut être vu comme une montée en généralité (Boltanski et Thévenot, 1991 ; Lafaye et Thevenot, 1993) pour répondre à des exigences d'universalisation et s'assurer de la portée d'une cause (Chateauraynaud, 2007). La défense de l'agriculture biologique peut aussi permettre de privilégier certaines « ouvertures d'avenir » (Chateauraynaud et Doury, 2011) comme l'avènement d'une société prenant en compte les enjeux environnementaux ou prévenir des «prophéties de malheur » (Chateauraynaud et Torny, 1999) telles que l'épuisement des ressources pétrolières, argument effectivement entendu dans nos entretiens. Ce mode de production est souvent vu par le grand public comme celui respectant au mieux l'avenir environnemental de la planète. Enfin, l'engagement en faveur de l'agriculture biologique peut permettre de rallier un bon nombre d'acteurs impliqués dans la défense de l'environnement au niveau local, national comme au niveau international et ainsi constituer une sorte de contrepouvoir (Rosanvallon, 2006) ${ }^{6}$. En ce sens,

6. Cela a également été montré dans l'analyse des controverses autour des OGM, la cause de l'agriculture biologique servant d' " argument de convergence aux faucheurs pour étendre la mobilisation » (Chateauraynaud, Bernard de Raymond et al., 2010). la défense de l'agriculture biologique et l'alliance avec des acteurs de la société civile spécialisés dans le domaine environnemental peuvent apparaître comme un moyen de résistance à distance au pouvoir en place, comme c'est le cas dans deux de nos terrains étudiés, où des projets d'aménagements sont soutenus par certains acteurs des pouvoirs publics.

On voit ainsi que l'agriculture biologique peut-être constituée comme un outil et un argument pour promouvoir une certaine vision de la société. Sa défense fonctionne comme un argument d'autorité qui peut difficilement être contré et entravé et les acteurs en sont conscients. Ainsi, dans le cas des Yvelines, où on a pu observer un conflit entre un projet de construction d'un circuit de Formule 1 et l'installation d'exploitations engagées à produire selon le cahier des charges de l'agriculture biologique ; le collectif mobilisé contre le projet de circuit de Formule 1 a adopté une stratégie d'interpellation dans les médias des pouvoirs publics et des élus locaux et nationaux, en leur demandant de prendre position « pour ou contre le projet de circuit de Formule $1 »-$ sous-entendu «pour ou contre l'agriculture biologique ». Cette affaire se déroulant dans le contexte de l'élaboration des lois Grenelle 1 et 2 fixant les mesures à prendre pour une meilleure protection de l'environnement et de la santé, la majorité des acteurs s'étant exprimés publiquement a pris position contre la construction du projet de Formule 1 en évoquant l'importance des enjeux environnementaux. Le projet a finalement été abandonné.

La diffusion et le succès de l'agriculture biologique conduisent donc d'une certaine façon à une forme d'instrumentalisation de ce mode de production dans certaines actions collectives. Se pose alors la question des résultats d'une telle utilisation : est-ce là un simple procédé rhétorique ou peut-on noter des effets concrets ? 


\section{L'agriculture biologique, aiguillon pour une société soucieuse des enjeux environnementaux}

De fait, l'argument du développement de l'agriculture biologique ne se limite pas à être un "opérateur de convergences de bonnes causes » (Chateauraynaud, Bernard de Raymond et al., 2010), mais permet effectivement le développement de ce mode de production et plus globalement de pratiques respectueuses de l'environnement.

Dans le cas étudié se situant dans les Yvelines, après que le projet de circuit de Formule 1 a été abandonné, deux maraîchers et un céréalier biologiques se sont installés avec des projets de vente directe et d'approvisionnement de la restauration collective locale. Leurs installations ont depuis donné lieu à la création de la première légumerie biologique ${ }^{7}$ d'Îlede-France inaugurée en mars 2012. Par ailleurs, la mairie des Mureaux s'est vu attribuer deux parcelles d'environ 4 hectares chacune pour des jardins familiaux et des ruchers. Le Syndicat des Berges de la Seine et de l'Oise a récupéré une bande de terrain en bordure de Seine pour la création d'un chemin de halage. Enfin, le Conseil général des Yvelines a, lui, demandé une bande de 8 mètres de large à proximité de la route départementale 14 pour aménager une piste cyclable. Dans un autre des terrains observés, en Essonne, la mobilisation d'acteurs pour le développement d'une agriculture plus «propre » pour la préservation de leur cadre de vie a conduit à la

7. Une légumerie biologique est un atelier destiné à la préparation élémentaire (lavage, épluchage, découpage...) des légumes biologiques à destination des cuisines de la restauration collective qui sont souvent dépourvues de ce genre d'atelier. Elle permet de centraliser le traitement de légumes produits à proximité et peut donc favoriser l'approvisionnement de la restauration collective en produits locaux. création d'une $\mathrm{AMAP}^{8}$ dont les cultures sont conduites en agriculture biologique, pour légitimer la préservation d'un espace agricole. Deux cent cinquante familles et plusieurs agriculteurs sont en partenariat au sein de ce dispositif. L'un des agriculteurs est un céréalier dont l'exploitation se situe à proximité du lieu de vie des habitants à l'origine de l'initiative. Au commencement du projet, cet agriculteur cultivait uniquement des céréales en agriculture conventionnelle sur un espace menacé d'urbanisation. Aujourd'hui, il a converti la totalité de son exploitation à l'agriculture biologique et diversifié sa production (lentilles, pommes de terre...). Il a aussi permis la création d'emplois et d'exploitations. En effet, un atelier de boulangerie a été mis en place sur sa ferme et un volailler biologique s'est installé sur une partie de ses terres. Le recours à l'argument du développement de l'agriculture biologique dans des mobilisations collectives n'est pas seulement une stratégie rhétorique, cela peut aussi avoir des conséquences en termes d'écologisation de l'agriculture ou d'un territoire.

Par ailleurs, les mobilisations collectives en faveur du développement de l'agriculture biologique peuvent aussi avoir des effets sur les relations entre monde agricole et monde non agricole. Lorsque des acteurs non agricoles se mobilisent pour le développement de l'agriculture

8. AMAP : «Association pour le maintien d'une agriculture paysanne ayant pour objectif de préserver l'existence et la continuité des fermes de proximité dans une logique d'agriculture durable [...]. Elle réunit un groupe de consommateurs et un agriculteur de proximité autour d'un contrat dans lequel chaque consommateur achète en début de saison une part de la production qui lui est livrée périodiquement à un coût constant. Le producteur s'engage à fournir des produits de qualité dans le respect de la charte de l'agriculture paysanne » (Extrait de la Charte des AMAP, mai 2003, disponible sur : www.reseau-amap.org/ docs/chartedesamap.PDF). 
biologique, les prémisses de la mobilisation peuvent s'avérer conflictuelles, car cette mobilisation peut être perçue par les agriculteurs conventionnels comme une forme de critique de leur activité. Cela a notamment été le cas sur les terrains observés en Essonne ou en Seine-et-Marne. Néanmoins, on a observé que des apprentissages entre acteurs du monde non agricole et acteurs agricoles pouvaient avoir lieu : apprentissages du côté des acteurs non agricoles sur l'histoire du modèle agricole « intensif » issu de la modernisation agricole, sur les contraintes économiques des exploitations en grandes cultures, sur les problèmes de débouchés... Et aussi apprentissages du côté des agriculteurs, sur ce qu'est l'agriculture biologique et sur certaines conceptions de la protection de la planète et de la biodiversité... Si ce partage de connaissances ne va pas toujours de soi, comme le montrent les propos de cet agriculteur, des compromis peuvent être néanmoins effectués.

"C'est compliqué, mettre en rapport des gens qui n'ont pas du tout la même vue d'esprit sur ce que c'est que le développement durable, ce que c'est que l'agriculture biologique, ce que c'est que le développement... la défense.... la protection de la planète...» (Agriculteur conventionnel).

En effet, à travers des interactions fréquentes, acteurs agricoles et acteurs non agricoles mobilisés en faveur du développement de l'agriculture biologique peuvent parvenir à élaborer des propositions préservant leurs intérêts parfois divergents. Ainsi, en Seine-et-Marne, on a pu voir qu'en plus de proposer l'agriculture biologique, les acteurs mobilisés pour la protection de l'eau se sont attachés à proposer également aux agriculteurs toute une panoplie de "pratiques intermédiaires ». Ces pratiques ne nécessitent pas une totale reconception du système de production et pourtant devraient permettre l'amélioration de la qualité de l'eau. En
Essonne, les acteurs mobilisés pour la protection de leur cadre de vie souhaitaient en premier lieu proposer aux agriculteurs de l'espace menacé d'urbanisation de convertir leurs exploitations à l'agriculture biologique, notamment en vue d'approvisionner la restauration collective locale. Devant l'opposition d'un certain nombre d'entre eux, des discussions se sont engagées, des arguments ont été échangés et c'est finalement l'idée de la mise en place d'une agriculture « propre »- basée sur une réduction de l'usage des intrants de synthèse et la réduction des transports entre le lieu de production et de livraison (les lieux de restauration collective à proximité) - qui a été adoptée. Ainsi, on voit se construire des formes de dialogues et d'ajustements entre acteurs agricoles et acteurs non agricoles.

Enfin, sur la base de l'instauration de ce dialogue, les mobilisations collectives en faveur de l'agriculture biologique peuvent également permettre d'envisager d'autres formes d'organisation de la société. Par exemple à travers la mise en place de systèmes d'approvisionnement de la restauration collective locale grâce à l'agriculture de proximité, comme dans le cas que l'on vient d'évoquer ou dans le cas des Yvelines à la suite de l'abandon du projet de circuit de Formule 1. En effet, ce type de dispositif permet de multiplier les interactions entre acteurs agricoles et acteurs non agricoles, de favoriser leur intercompréhension et de créer de nouvelles interdépendances entre eux. Dans une perspective plus théorique, on peut également penser que le fait que des acteurs non agricoles se mobilisent pour le développement d'un mode de production permettant de préserver, par exemple, l'eau ou le paysage peut promouvoir certaines formes de gestion collective. Effectivement, dans les cas observés, des acteurs non agricoles se mobilisent pour l'agriculture biologique, s'investissent dans l'accompagnement des agriculteurs pour favoriser leurs changements de pratiques et font souvent appel 
RECHERCHES

Aurélie CARDONA

aux acteurs publics (élus régionaux, départementaux ou municipaux) pour soutenir leur action. En ce sens, on peut considérer qu'ils envisagent l'eau ou le paysage, comme des «biens communs » dont la préservation passe par une gestion collective et concertée (Ostrom, Burger et al., 1999). Cette perspective peut s'avérer tout à fait innovante dans notre société où la tendance majoritaire est à la privatisation, comme le montrent les discussions autour de la rémunération des services écosystémiques produits par l'agriculture.

Lorsque les acteurs non agricoles investissent le domaine de l'agriculture biologique, cela peut avoir des effets tangibles sur l'agriculture et les territoires. Ainsi, la diffusion de l'agriculture biologique et le fait que des acteurs non agricoles s'en saisissent de manière originale, a un certain pouvoir transformateur à l'échelle de la société dans son ensemble constituant un terreau fertile pour l'élaboration de formes d'organisationnelles innovantes.

$$
\begin{gathered}
* \\
* *
\end{gathered}
$$

$\mathrm{Au}$ vu de notre analyse, le développement de l'agriculture biologique ne doit pas uniquement être considéré sous l'angle de la diffusion d'un mode de production particulier. La perception qu'en a le monde agricole évolue, car il est mieux connu, mais aussi en raison de l'affaiblissement de son ressort contestataire qui entretenait une opposition radicale entre agriculture conventionnelle et agriculture biologique. Désormais, il est davantage vu comme un mode de production parmi d'autres, dans lequel les agriculteurs peuvent piocher pour «bricoler » de nouveaux itinéraires techniques moins consommateurs en intrants de synthèse, plus économiques et potentiellement plus respectueux de l'environnement. En ce qui concerne le monde non agricole, l'agriculture biologique apparaît comme un nouvel outil et argument permettant de défendre des causes diverses. Prôner le développement de l'agriculture biologique peut en effet être utilisé pour valoriser un espace menacé d'urbanisation ou encore pour préserver des biens communs tels que l'eau. En outre, ces mobilisations d'acteurs non agricoles en faveur du développement de l'agriculture biologique semblent pouvoir être à l'origine de propositions originales en termes organisationnels, que cela soit en recréant des interdépendances fortes entre monde agricole et monde non agricole ou en envisageant des formes de gestions collectives pour certains biens à préserver.

Ainsi, une diversité d'acteurs participent au développement de l'agriculture biologique, s'en inspirent, la mettent en variation, s'en détournent - ce qui peut donner lieu à des pratiques et des propositions organisationnelles innovantes impliquant acteurs agricoles et acteurs non agricoles. Ce constat est particulièrement intéressant, car il ouvre un pan de recherche visant à comprendre le rôle que peuvent jouer les acteurs non agricoles dans les processus de transitions vers des formes d'agricultures plus respectueuses de l'environnement.

Cet article a été largement amélioré par les suggestions de relecteurs que je souhaite remercier. Cette recherche a été soutenue par l'attribution d'une allocation doctorale de la Région Île-deFrance et par le projet ANR POPSY (Systèmes de production de grandes cultures, environnement, politiques publiques). 


\section{RÉFÉRENCES BIBLIOGRAPHIQUES}

Ansaloni M., Fouilleux E. (2006). Changements de pratiques agricoles : facteurs et modalités d'hybridation technique des exploitations laitières bretonnes. Économie rurale, 292, p. 3-17.

Bessy C., Chateauraynaud F. (1995). Experts et faussaires. Pour une sociologie de la perception, Paris, Metailié, 360 p.

Boltanski L., Thévenot L. (1991). De la justification : les économies de la grandeur. Paris, Gallimard, 483 p.

Cardona A. (2012). L'agriculture à l'épreuve de l'écologisation. Éléments pour une sociologie des transitions, Thèse de sociologie, EHESS, 429 p.

Cardona A., Lamine C., Hochereau F. (2012). Mobilisations et animations autour des réductions d'intrants : stratégies d'intéressement des agriculteurs dans trois territoires franciliens. Revue d'Études en Agriculture et Environnement, $\mathrm{n}^{\circ}$ 93-1, p. 49-70.

Chateauraynaud F. (2007). Des disputes ordinaires à la violence politique. L'analyse des controverses et la sociologie des conflits. Communication au Colloque Types de conflits et formes de politisation, Université du Maine, 13 p.

Chateauraynaud F., Bernard de Raymond A., Hermitte M.-A., Tetart G. (2010). Les OGM entre régulation économique et critique radicale. Rapport du programme OBSOGM, ANR OGM, GSPR, Paris, 420 p. Disponible sur : http://gspr.ehess.free.fr/documents/ rapports/RAP-2010-ANROGM.pdf

Chateauraynaud F., Doury M. (2011). La portée des précédents. Événements marquants et procédés argumentatifs. Socio-informatique et argumentation [en ligne], Hypothèse. org. Disponible sur : http://socioargu.hypotheses.org/2274

Chateauraynaud F., Torny D. (1999). Les Sombres précurseurs. Une sociologie pragmatique de l'alerte et du risque. Paris, EHESS, 476 p.

Guthman J. (2004). The trouble with "organic lite" in California: a rejoinder to the "conventionalisation" debate. Sociologia ruralis, vol. 44, n³, p. 301-316.

Hill S. B., MacRae R. J. (1996). Conceptual framework for the transition from conventional to sustainable agriculture. Journal of Sustainable Agriculture, $\mathrm{n}^{\circ}$ 7, p. 81-87.

Lafaye C., Thévenot L. (1993). Une justification écologique ? Conflits dans l'aménagement de la nature. Revue francaise de sociologie, vol. 34, $\mathrm{n}^{\circ}$ 4, p. 495-524.

Lamine C. (2011). Anticiper ou temporiser. Injonctions environnementales et recompositions des identités professionnelles en céréaliculture. Sociologie du travail, $\mathrm{n}^{\circ} 53$, p. 75-92.

Lamine C., Bellon S. (2009). Conversion to organic farming: a multidimensional research object at the crossroads of agricultural and social sciences. Agronomy for Sustainable Development, $\mathrm{n}^{\circ}$ 29, p. 97-112.

Le Pape Y., Rémy J. (1988). Agriculture biologique: unité et diversité. In Jollivet M., Pour une agriculture diversifiée (p. 134-140), Paris, L'Harmattan.

Leroux B. (2011). Les agriculteurs biologiques et l'alternative. Contribution à l'anthropologie politique d'un monde paysan en devenir. Thèse de Sociologie, CSE, EHESS, 409 p.

Mélard F. (2008). Écologisation. Objets et concepts intermédiaires. Bruxelles, Peter Lang, 214 p.

Michelsen J. (2001). Recent Development and Political Acceptance of Organic Farming in Europe. Sociologia ruralis, $\mathrm{n}^{\circ}$ 41, p. 3-20.

Ostrom E., Burger J., Field C. B., Norgaard R. B., Policansky D. (1999). Revisiting the commons: local lessons, global challenges. Science, $\mathrm{n}^{\circ} 284$, p. 278-282.

Piriou S. (2002). L'institutionnalisation de l'agriculture biologique (1980-2000). Thèse de l'ENSAR, ENSAR, 461 p.

Remy J. (1989). De la glèbe au substrat : agriculteurs biologiques et maraîchers horssol. In Mathieu N., Jollivet M., Du rural à l'environnement : la question de la nature aujourd'hui (p. 123-146), Paris, ARF/ L'Harmattan.

Rosanvallon P. (2006). La contre-démocratie: la politique à l'âge de la défiance. Paris, Seuil, 344 p.

Van Dam D., Nizet J., Dejardin M., Streith M. (2009). Les agriculteurs biologiques : ruptures et innovations. Dijon, Educagri, 141 p. 